\title{
Alkylamine Integrated Metal-Organic Framework-Based Waveguide Sensors for Efficient Detection of Carbon Dioxide from Humid Gas Streams
}

Ki-Joong Kim, ${ }^{,+, \neq}$Jeffrey T. Culp, ${ }^{\dagger \neq}$ Paul R. Ohodnicki, ${ }^{\dagger}$ Patricia C. Cvetic, ${ }^{\dagger, \ddagger}$ Sean

Sanguinito, ${ }^{+\neq}$Angela L. Goodman, ${ }^{\dagger}$ and Hyuk Taek Kwon ${ }^{\S}$

${ }^{\dagger}$ National Energy Technology Laboratory, 626 Cochrans Mill Road, Pittsburgh, Pennsylvania 15236, United States

${ }^{\ddagger}$ Leidos Research Support Team, 626 Cochrans Mill Road, Pittsburgh, Pennsylvania 15236, United States

${ }^{\S}$ Department of Chemical Engineering, Pukyong National University, 45 Yongso-ro, Nam-gu, Busan 48513, South Korea

Corresponding author: ki-joong.kim@netl.doe.gov 
Table of contents:

Figure S1. (a) $\mathrm{N}_{2}$ adsorption isotherms and (b) pore size distributions of Co/ZIF8 and OLA-Co/ZIF8 samples.

Figure S2. TGA results of OLA, Co/ZIF8 and OLA-Co/ZIF8 samples.

Figure S3. Optical spectra of OLA-Co/ZIF8 coated optical fiber sensor under $\mathrm{N}_{2}$ and $\mathrm{CO}_{2}$ flow.

Figure S4. Breakthrough curves for $\mathrm{H}_{2} \mathrm{O}$ of Co/ZIF8 and OLA-Co/ZIF8 samples with and without $\mathrm{CO}_{2}$.

Figure S5. Adsorbed amount of $\mathrm{H}_{2} \mathrm{O}$ on Co/ZIF8 and OLA-Co/ZIF8 samples, calculated from breakthrough curves in Figure S3.

Figure S6. (a) $\mathrm{CO}_{2}$ adsorption isotherm in dry condition and (b) comparing mass gain in samples of Co/ZIF8 and OLA-Co/ZIF8 under dry $\mathrm{N}_{2}$, humid $\mathrm{N}_{2}$, and humid $\mathrm{CO}_{2}$.

Figure S7. Temperature programmed desorption results. (a) Desorption characteristics and (b, c) desorbed amount of $\mathrm{CO}_{2}$ and $\mathrm{H}_{2} \mathrm{O}$ from Co/ZIF8 and OLA-Co/ZIF8 samples, calculated from Figure S6a.

Figure S8. (a) Photographs and (b) XRD patterns of as-synthesized Co/ZIF8 and OLA-Co/ZIF8 in the mixture of water and hexane at different times.

Figure S9. FT-IR spectra of the Co/ZIF8 and OLA-Co/ZIF8 after long air exposures.

Figure S10. In-situ FT-IR spectra of the (a) Co/ZIF8 and (b) OLA-Co/ZIF8 in the region of adsorbed water under the flow of humid flue gas at $25^{\circ} \mathrm{C}$ under $32 \mathrm{psi} \mathrm{CO}_{2}$ with increasing $\mathrm{RH}$. 


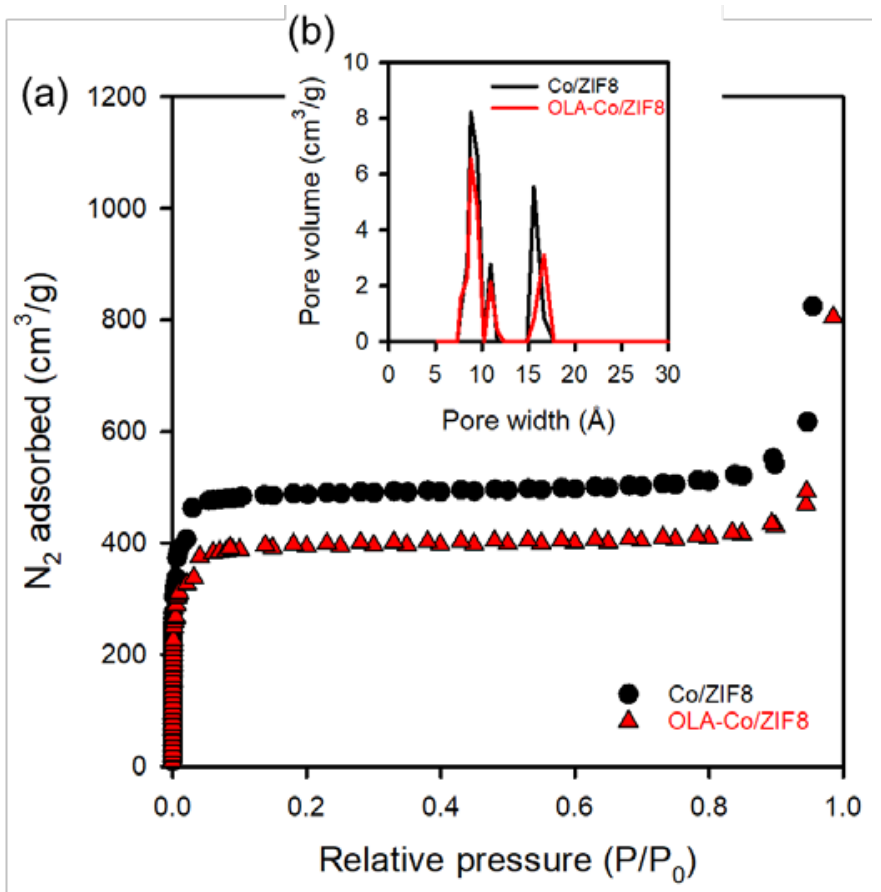

Figure S1. (a) $\mathrm{N}_{2}$ adsorption isotherms and (b) pore size distributions of Co/ZIF8 and OLA-Co/ZIF8 samples.

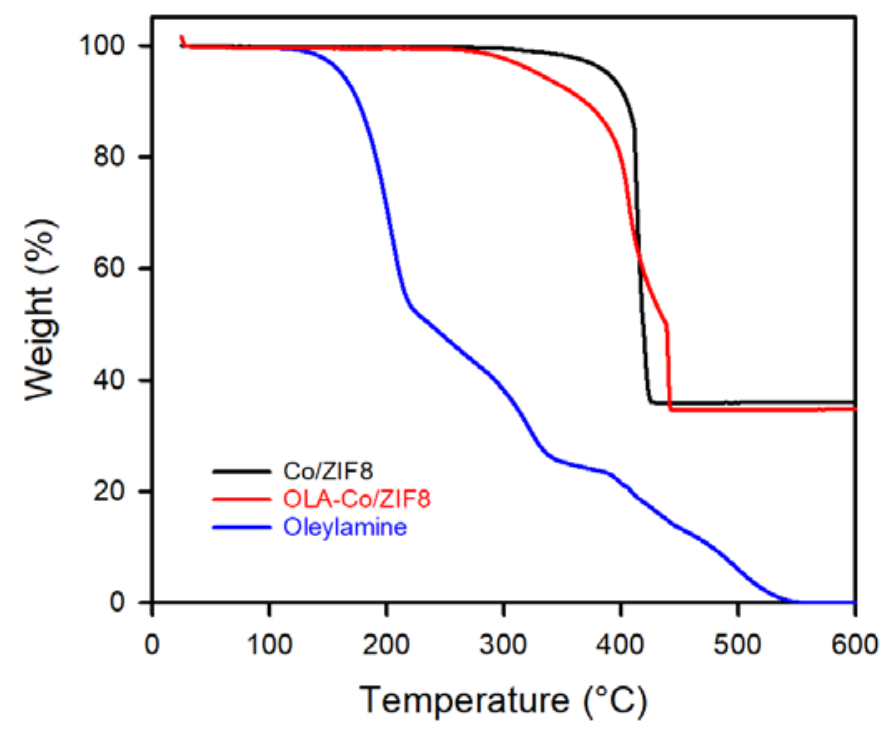

Figure S2. TGA results of OLA, Co/ZIF8 and OLA-Co/ZIF8 samples. 


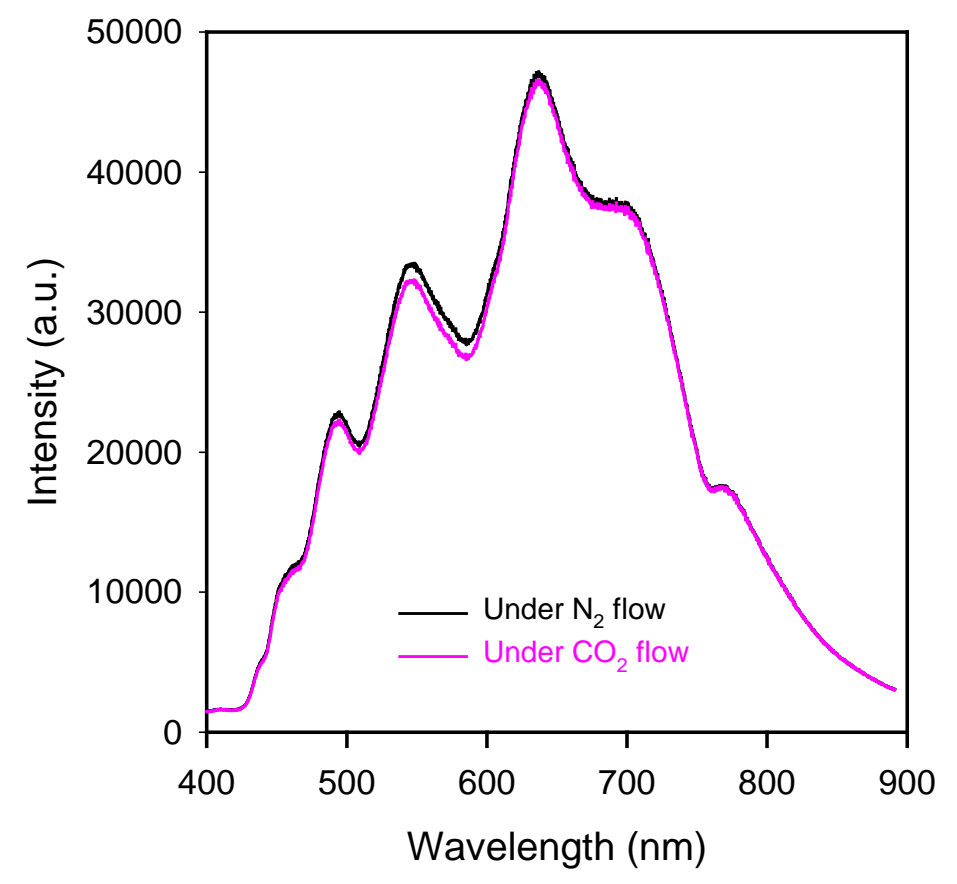

Figure S3. Optical spectra of OLA-Co/ZIF8 coated optical fiber sensor under $\mathrm{N}_{2}$ and $\mathrm{CO}_{2}$ flow.

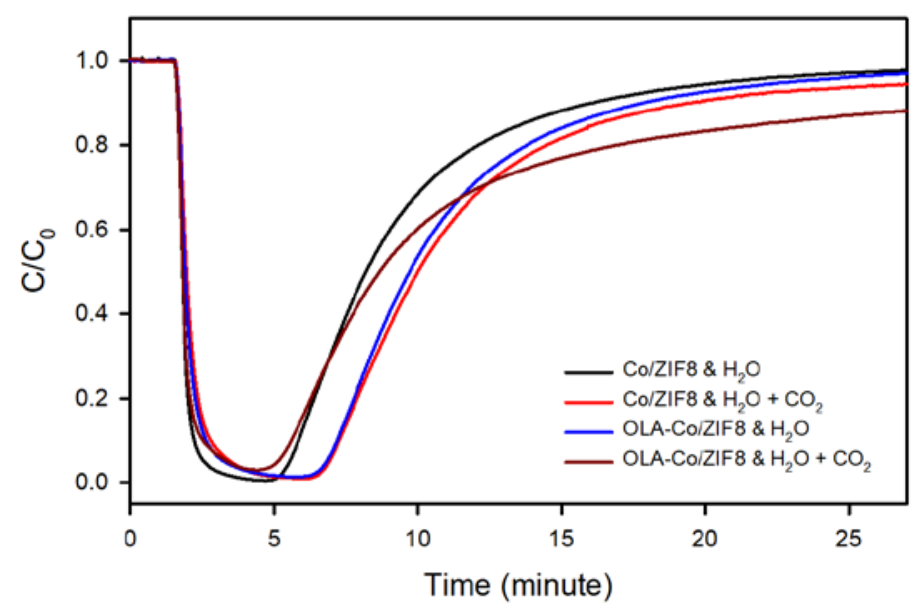

Figure S4. Breakthrough curves for $\mathrm{H}_{2} \mathrm{O}$ of Co/ZIF8 and OLA-Co/ZIF8 samples with and without $\mathrm{CO}_{2}$. 


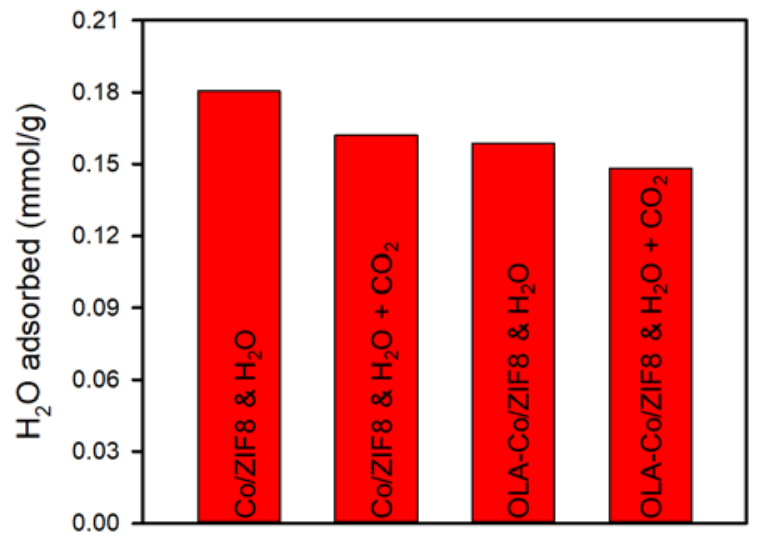

Figure S5. Adsorbed amount of $\mathrm{H}_{2} \mathrm{O}$ on Co/ZIF8 and OLA-Co/ZIF8 samples, calculated from breakthrough curves in Figure $S 4$.
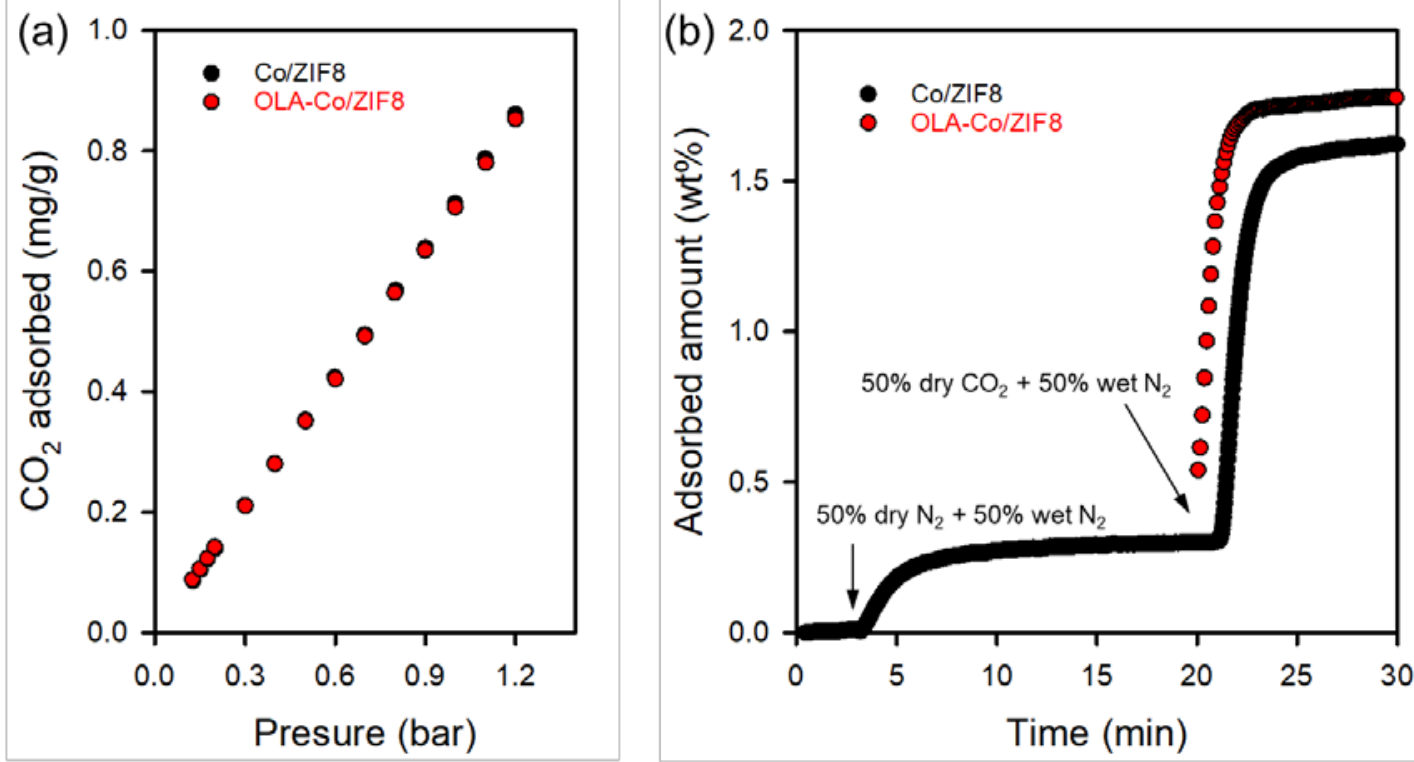

Figure S6. (a) $\mathrm{CO}_{2}$ adsorption isotherm in dry condition and (b) comparing mass gain in samples of $\mathrm{Co} /$ ZIF8 and OLA-Co/ZIF8 under dry $\mathrm{N}_{2}$, humid $\mathrm{N}_{2}$, and humid $\mathrm{CO}_{2}$. 

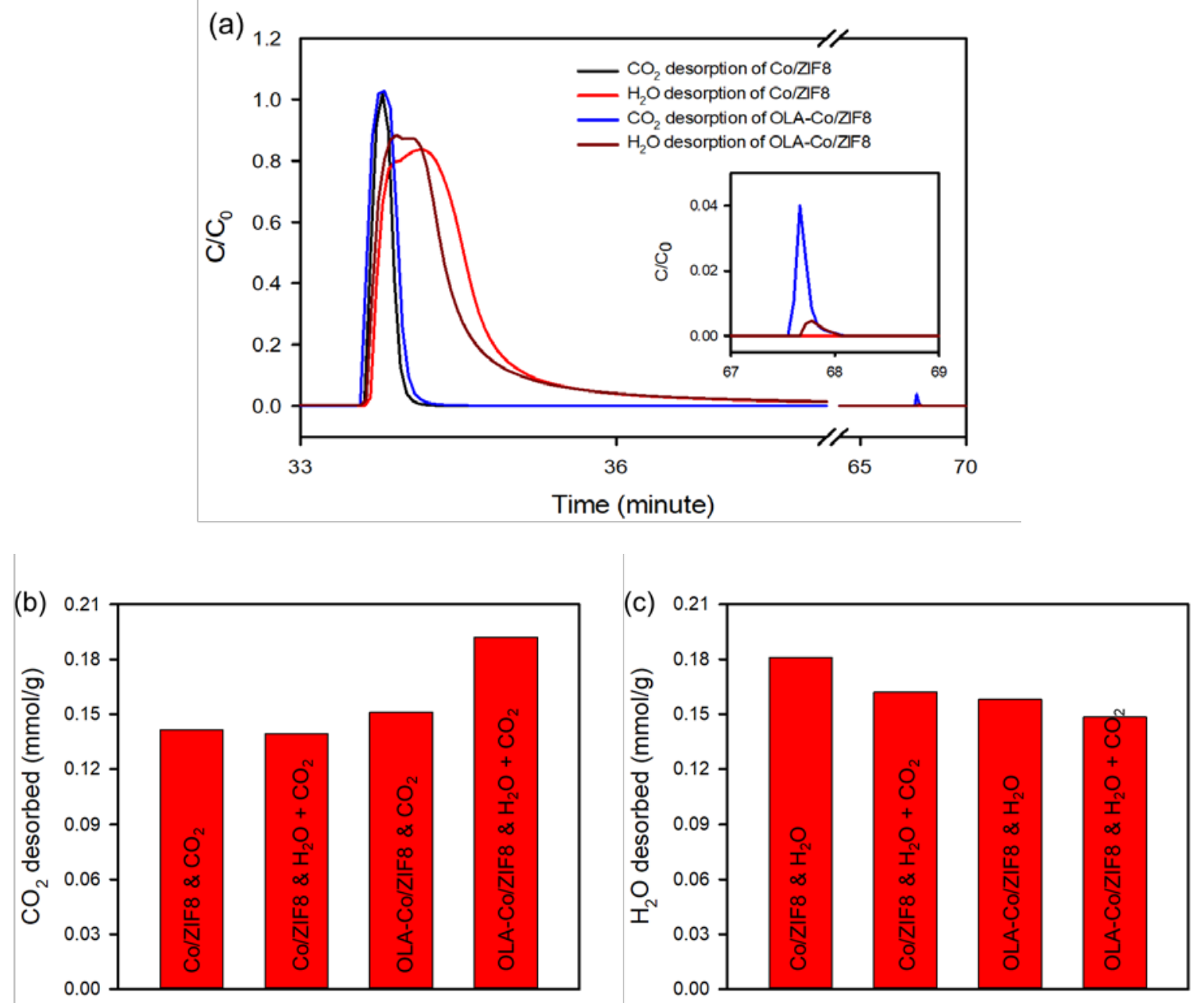

Figure S7. Temperature programmed desorption results. (a) Desorption characteristics and (b, c) desorbed amount of $\mathrm{CO}_{2}$ and $\mathrm{H}_{2} \mathrm{O}$ from Co/ZIF8 and OLA-Co/ZIF8 samples, calculated from Figure S7a. 

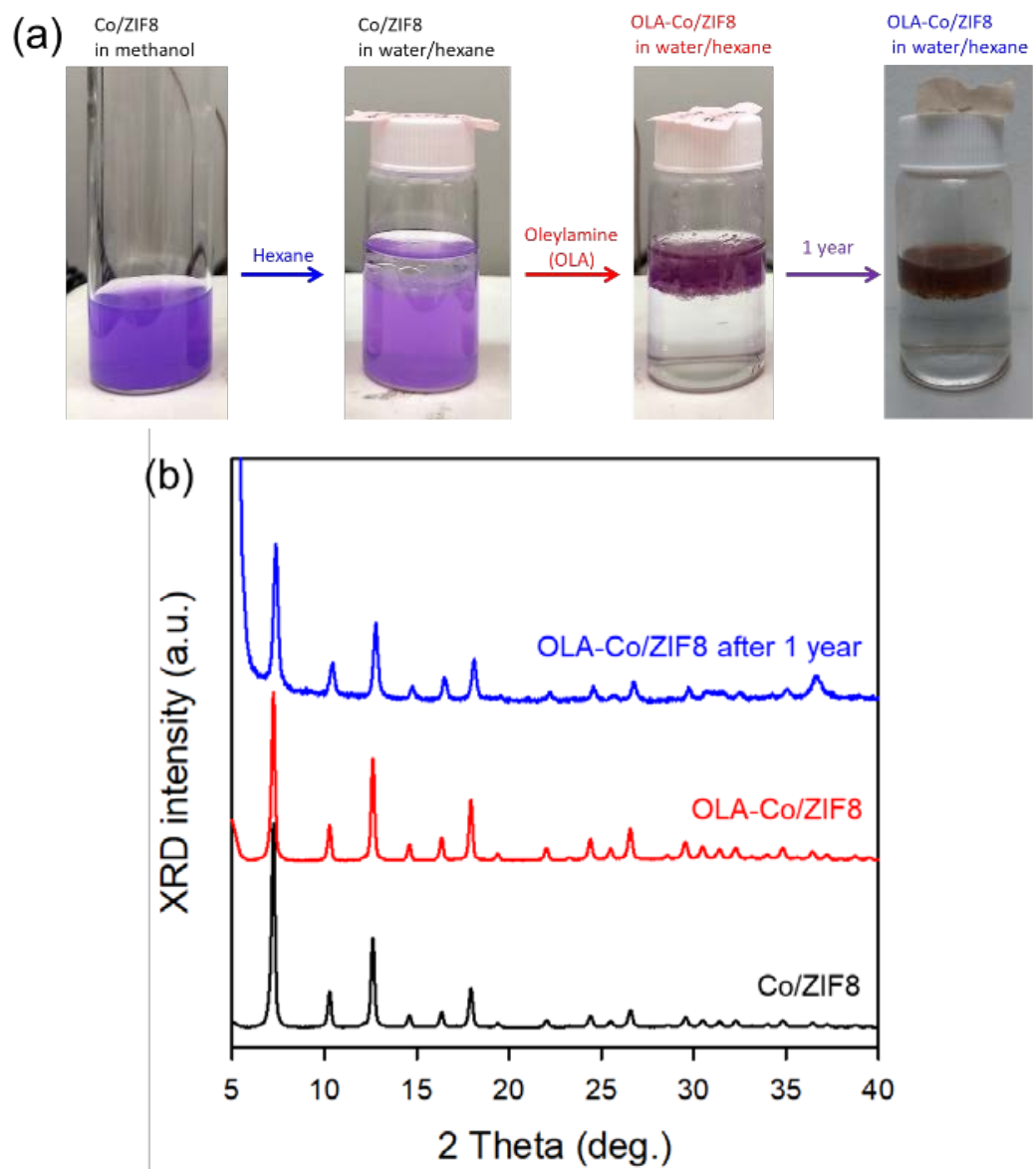

Figure S8. (a) Photographs and (b) XRD patterns of as-synthesized Co/ZIF8 and OLA-Co/ZIF8 in the mixture of water and hexane at different times. The samples were collected by centrifuge and dried for $\mathrm{XRD}$ analysis. 


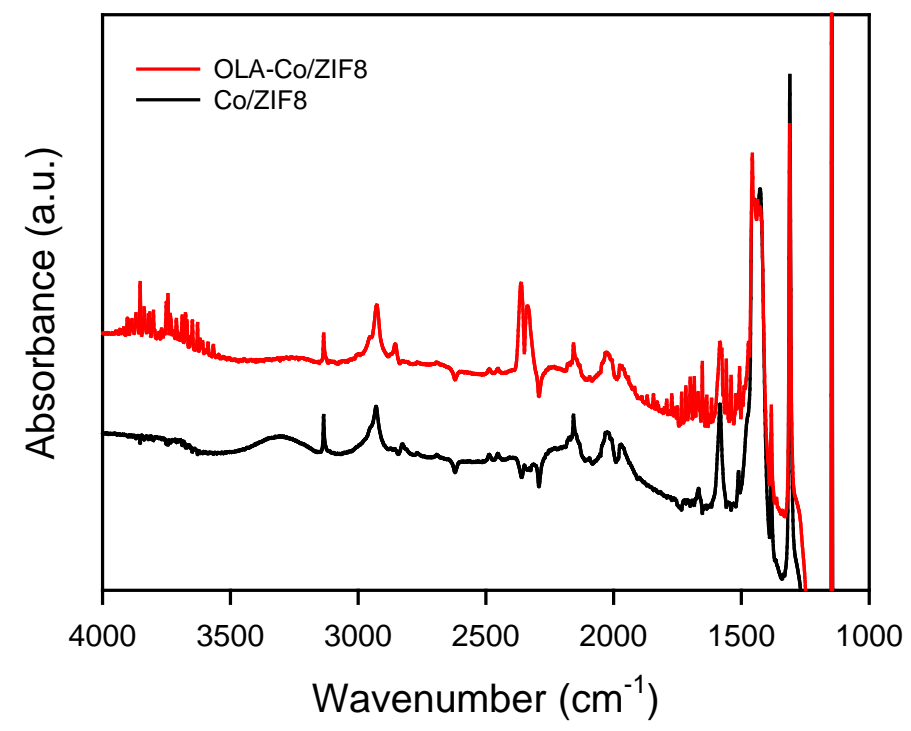

Figure S9. FT-IR spectra of the Co/ZIF8 and OLA-Co/ZIF8 after long air exposures. In the OH stretching region (3000 $4000 \mathrm{~cm}^{-1}$ ), the gas phase water molecule has a very small moment of inertia on rotation which gives vibrational rotational spectra containing tens of thousands of absorption lines. In the liquid, rotations tend to be restricted by hydrogen bonds, giving the libations. Also, spectral lines are broader causing overlap of many of the absorption peaks.
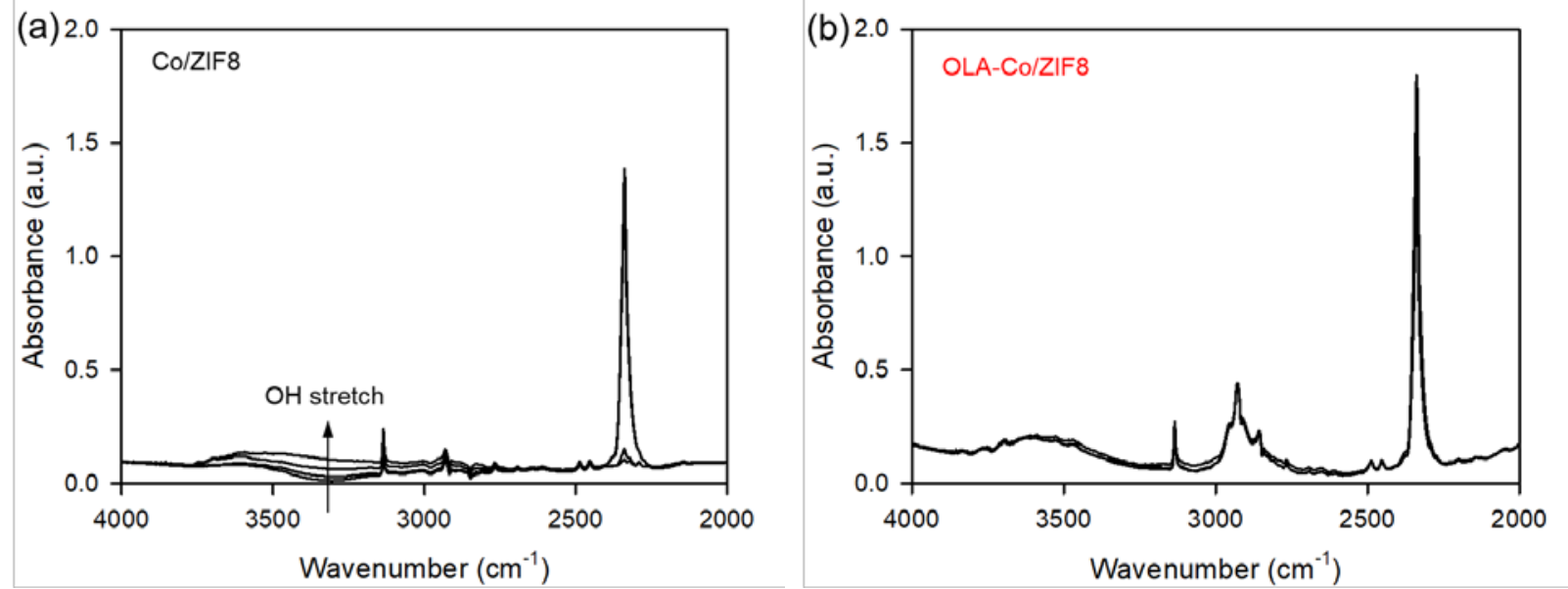

Figure S10. In-situ FT-IR spectra of the (a) Co/ZIF8 and (b) OLA-Co/ZIF8 in the region of adsorbed water under the flow of humid flue gas at $25^{\circ} \mathrm{C}$ under $32 \mathrm{psi} \mathrm{CO}_{2}$ with increasing $\mathrm{RH}$. 